\title{
SI00A4 in Spinal Substantia Gelatinosa from Dorsal Root Ganglia Modulates Neuropathic Pain in a Rodent Spinal Nerve Injury Model
}

This article was published in the following Dove Press journal:

Journal of Pain Research

\author{
Xin Jiang $\mathbb{B D}^{1, *}$ \\ Wenqi Zhao',* \\ Tiantian Zhao $\mathbb{D}^{2, *}$ \\ Mei Yang' \\ Hongbin Yuan' \\ Jun Qian ${ }^{2}$ \\ Zhenghua Xiang ${ }^{3}$ \\ 'Department of Anesthesiology, \\ Changzheng Hospital, Naval Medical \\ University, Shanghai, People's Republic of \\ China; ${ }^{2}$ Department of General Surgery, \\ Affiliated Xinchang Hospital of Shaoxing \\ University, Zhejiang, People's Republic of \\ China; ${ }^{3}$ Department of Neurobiology, Key \\ Laboratory of Molecular Neurobiology, \\ Ministry of Education, Naval Medical \\ University, Shanghai, People's Republic of \\ China \\ *These authors contributed equally to this \\ work
}

Purpose: To detect the spatio-temporal expression of S100A4 in a spinal nerve ligation (SNL) rat model. Also to figure out which other molecules directly interact with S100A4 to explore the possible mechanisms which might be involved in neuropathic pain.

Methods: Seven-week-old male SD rats were used for the SNL model construction. Immunofluorescence and Western blotting were used to detect the spatio-temporal expression of S100A4 in the model. S100A4 was co-labeled with a number of related molecules and marker molecules that can distinguish between cell types. After intrathecal injection of S100A4 neutralizing antibody, the behavioral changes of SNL rats were recorded, and molecular changes compared. The direct interaction between S100A4 and other related molecules was verified by co-immunoprecipitation (co-IP) to explore its possible mechanism.

Results: After spinal nerve ligation, the content of S100A4 in the dorsal root ganglion (DRG) and spinal dorsal horn increased significantly. Intrathecal injection of S100A4 neutralizing antibody could effectively relieve the mechanical pain in rats. co-IP revealed a direct interaction between S100A4 and RAGE.

Conclusion: The content of S100A4 in the DRG and spinal dorsal horn of SNL rats increased, compared with that of the control group. Intrathecal injection of S100A4 neutralizing antibody could effectively relieve the mechanical pain in SNL rats. S100A4 may be involved in the production of neuropathic pain through RAGE or other ways, but the specific mechanism needs to be further studied.

Keywords: neuropathic pain, S100 calcium binding protein A4, receptor for advanced glycation end products, spinal nerve ligation model

\section{Plain Language Summary}

Although the mechanism of neuropathic pain is complex, the role of inflammatory mediators in it is widely recognized. S100A4 is highly expressed in sites of neurogenesis and lesions, indicating a role in neuronal plasticity. In this study, seven-week-old male SD rats were used to make SNL model. Immunofluorescence and Western blotting were used to detect the spatio-temporal expression of S100A4 in the model. S100A4 was co-labeled with a number of related molecules and marker molecules that can distinguish between cell types. Pain was reduced by intrathecal injection of S100A4 neutralizing antibody. The behavioral changes of rats were recorded, and molecular changes compared. The direct interaction between S100A4 and other related molecules was verified by co-IP in order to explore its possible mechanism. The results showed that the content of S100A4 in the DRG and spinal dorsal horn increased, and that slow intrathecal injection of S100A4 neutralizing antibody could effectively relieve the mechanical pain in rats, indicating that S100A4 may be involved in the production of neuropathic pain through RAGE or other ways.
Jun Qian

Department of General Surgery, Affiliated Xinchang Hospital of Shaoxing University, No. 117 Middle Gushan Road, Xinchang, Zhejiang, 3/2500, People's Republic of China

Tel +865758638079

Email qianj00I@I63.com
Journal of Pain Research 2021:|4 665-679

submit your manuscript DovePress f $y$ in

http://doi.org/10.2147/JPR.\$293462 


\section{Introduction}

Neuropathic pain, a persistent and intractable problem worldwide, refers to pain caused by pathological changes or diseases of the somatosensory nervous system. The existence of neuropathic pain increases the disease burden of people with chronic pain, which is mainly manifested as the decline of quality-of-life, deterioration of health status, difficulties in employment, and heavy medical expenses. ${ }^{1}$ Neuropathic pain starts as an adverse adaptive reaction of the nervous system to injury, structure and function changes follow once the pathological changes of the sensory nervous system happen, stimulating neuropathic pain which is usually spontaneous. The response to harmful or harmless stimuli is pathologically magnified, making the pre-existing pain more severe or converting the nonnoxious stimulation to pain. Nerve injury is a necessary condition for neuropathic pain; however, it alone is not enough to produce neuropathic pain and is affected by other factors. ${ }^{2}$ The peripheral receptors transmit nociceptive stimuli into the dorsal horn of the spinal cord, with the neurons within being responsible for transmitting and modulating pain-related signals from the nociceptor through the spinal cord to the brain region. ${ }^{3}$ Neuropathic pain reflects the abnormal excitation of neurons in the dorsal horn of the spinal cord caused by peripheral sensory input, ${ }^{2}$ the formation of this abnormal excitation involves neurons, glial cells, and immune cells. Accumulating evidence shows that microglia in the spinal cord plays a key role in the origin of chronic pain by releasing proinflammatory cytokines, such as tumor necrosis factor $\alpha$ (TNF- $\alpha$ ), interleukin-1 $\beta$ (IL-1 $\beta$ ), and brain-derived neurotrophic factor (BDNF). ${ }^{4}$ In astrocytes, IL-1 $\beta$ treatment activated ERK and released MMP-2. ${ }^{5}$ The overexpression of oligodendrocyte-derived IL-33 at the spinal cord level is related to the release of other cytokines, including TNF- $\alpha$ and IL- $1 \beta .{ }^{6}$ In recent years, accumulating evidence suggests that the peripheral immune system and microglia are equally critical in inducing neuropathic pain. Cytokines and neutrophils take part in the early stages of acute pain, while $\mathrm{T}$ lymphocytes mainly play a central role in chronic neuropathic pain. ${ }^{7}$

S100 protein contains at least 25 members, forming the largest EF-hand signal proteome in human. ${ }^{8}$ After binding to $\mathrm{Ca}^{2+}, \mathrm{S} 100$ can quickly change their conformation and bind to the target protein to produce biological effects, thus, they are considered to be trigger proteins rather than buffer proteins, the latter maintaining a stable conformation to regulate $\mathrm{Ca}^{2+}$ concentration. ${ }^{9}$ S100A4 has many receptors related to inflammation-related signal transduction, such as advanced glycation end products receptor (RAGE), Toll-like receptor 4 (TLR4), and interleukin-10 receptor (IL-10R). ${ }^{10} \mathrm{~S} 100 \mathrm{~A} 4$ is highly expressed at sites of neurogenesis and lesions, indicating a role in neuronal plasticity. ${ }^{11}$ Some studies have shown that the neuroprotective effect of S100A4 depends partially on the increase of Akt signal transduction, which can reduce apoptosis. ${ }^{12}$ Extracellular S100A4 up-regulated several antioxidant enzymes and neuroprotective genes to protect nerve cells through the IL-10R/JAK/STAT3 pathway. ${ }^{13,14}$ S100A4 could also indirectly activate EGFR and induce neuroprotection through JAK/STAT, ERK, and Akt triggering signals. ${ }^{15,16}$ The increased expression of S100A4 in astrocytes after spinal cord transection indicated that the molecule takes part in the response of injured astrocytes. ${ }^{17}$ After the injury of dorsal root or sciatic nerve, the S100A4 in the dorsal functional area which experienced Wallerian degeneration was significantly up-regulated. ${ }^{18}$ Peripheral nerve or dorsal root injury induced a significant upregulation of S100A4 in the satellite cells of Schwann cells and ganglion at the injured site and distal stump. ${ }^{19}$

S100A4 is closely related to inflammatory response and nerve injury repair, both pathophysiological processes are the hot-spots of studies on neuropathic pain. Based on previous studies, we thought that S100A4 could be involved in neuropathic pain. A rat spinal nerve ligation (SNL) model was used to observe the expression of S100A4 in dorsal root ganglion and spinal dorsal horn, as well as the effect of intrathecal injection of S100A4 antibody on the behavior of model rats. The expression of S100A4-related molecules (RAGE, TLR4, and IL-10R) were detected to explore its possible molecular mechanism. Because credible results were only seen in RAGE, only results of experiments on interactions between RAGE and S100A4 were provided.

\section{Materials and Methods Experimental Animal}

7-week-old male Sprague Dawley (SD) rats were purchased from Shanghai Sippel-Bikai Company through the animal center and were raised in clean (CL) animal rooms with adequate food and water. Anesthesia and humanistic care were carried out during operations to minimize their suffering. 


\section{Ethics Statement}

All animal experiments were approved by the Animal Experiment Committee of Naval Medical University and performed in accordance with its Ethical and Safety Guidelines.

\section{Animal Experiments Measurement of Paw Withdrawal Threshold (PWT) in Rats $^{20}$}

The test environment was fixed in the same laboratory with the same light conditions, air conditioning temperature, and wind direction. Tests were conducted by the same person at $9 \mathrm{am}$. The rats to be tested were placed in a brown Plexiglas box with a wire mesh at the bottom, shaded at the top and adapted for 15 minutes. The finger web between the second and third toes of the left hindlimb was vertically stimulated by von Frey fiber (Stoelting, Wood Dale, US) from the gap of the bottom wire mesh. The strength of the fiber bending was $45^{\circ}$, the time was 10 seconds, and the stimulation interval was $\geq 30$ seconds. The selected fiber grams were $0.6,1,1.4,2,4,6$, $8,10,15$, and $26 \mathrm{~g}$. Foot withdrawal or foot licking reaction upon stimulation were recorded as positive, otherwise negative. This specific process was conducted according to the up-and-down method. ${ }^{20,21}$

\section{Building and Verification of Rat SNL Model}

The SNL model was built as described. ${ }^{9}$ Experimental rats were anesthetized with $1 \%$ pentobarbital solution $(40 \mathrm{mg} /$ $\mathrm{kg}$ ). The back skin was cut along the left side of the spine in the iliac crest plane, fascia cut layer by layer, muscles bluntly separated, the transverse process of No.6 lumbar removed by bone rongeur, and the lumbar 5 spinal nerve exposed. The lumbar 5 spinal nerve of the experimental group was ligated with 6-0 suture, while that of the control group (sham operation group) was only exposed for 5 minutes without ligation.

A successful model should present a PWT pain threshold which gradually reduces with time. Lost motor ability of the left hind-limb and no significant decrease in pain threshold in the absence of other interventions both mean failure of the model. Behavioral changes in the sham operation group suggests nerve injury failure of the sham operation.

\section{Construction and Verification of Intrathecal Catheters in Rats ${ }^{22,23}$}

After anesthesia, rats were cut along the mid-line of the spine at the iliac crest level, the lumbar 6 spinous process was removed, and the lumbar 5 inter-vertebral space exposed and cleaned. A PE-10 catheter filled with normal saline was inserted into the space towards the head side. Tail flick reaction meant successful placement of the catheter. The depth of the tube was about $1.5 \mathrm{~cm}$.

After the rat's mobility was restored, $10 \mu \mathrm{L}$ of $2 \%$ lidocaine was injected through the catheter, movement disturbance of the hind-limb indicating successful placement of the catheter within the sheath.

\section{Animal Grouping}

First, all rats were tested for pain threshold, those whose baseline mechanical PWT was $<26$ g were excluded. Fifteen rats with a qualifying threshold underwent SNL operation only, of which three rats were randomly chosen for tissue collection on the 3rd, 7th, 14th, 21st, and 28th day after operation.

Another 30 rats with a qualifying threshold were randomly divided into three groups ( $\mathrm{N}=10$ for each group): the control group, which underwent sham operation with intrathecal administration of normal saline; the SNL/salt group, which underwent SNL operation with intrathecal administration of normal saline; and the SNL/antibody group, which underwent SNL operation with intrathecal administration of S100A4 antibody. The intrathecal administration was given immediately after corresponding operation and repeated on the $1 \mathrm{st}, 3 \mathrm{rd}$, 5th, and 7th days thereafter. Then $0.2 \mu \mathrm{g}$ of S100A4 neutralizing antibody (mouse monoclonal IgG, Santa, USA) or the same volume of normal saline was injected. The total volume of intrathecal injection for each group was $15 \mu \mathrm{L}$. Injection was done slowly ( $3.3 \mu \mathrm{L} / \mathrm{min})$ to minimize the diffusion of the solution and confine its effect to mainly on the L5 segment of the spinal cord.

\section{Preparation of Tissue Sections}

After anesthesia, the left ventricle of rats was exposed and perfused with at least $250 \mathrm{~mL}$ saline until the effluent was clear and bloodless. Then $200 \mathrm{~mL}$ of $4 \%$ paraformaldehyde solution was injected until the torso and limbs became stiff. The lumbar 5 dorsal root ganglia and the spinal cord between the lumbar enlargement and sacral segment were collected and post-fixed with $4 \%$ paraformaldehyde for 4 to 6 hours, and then transferred to $25 \%$ sucrose solution for dehydration until the tissue samples sunk to the bottom of the tube. Samples were then embedded in O.T.C tissue freezing medium (SAKURA, America) and quickly frozen to $-20^{\circ} \mathrm{C}$ in a cryostat (Leica, Germany), cross-sectioned and floated in phosphate buffer. 
Each spinal cord sample was divided into six sections, which were placed into six-well plates in turn, and each section contained forty $20 \mu \mathrm{m}$ thickness slices. Each dorsal root ganglion samples was cut into $10 \mu \mathrm{m}$ thickness slices. Slices were affixed to the adhesive slide and dried in an oven at $37^{\circ} \mathrm{C}$ for 1 hour.

\section{Immunofluorescence}

In the SNL model, microglia in the spinal dorsal horn of the injured segments were activated. ${ }^{24}$ Ibal, a marker of microglial activation, was used to select the sections from the injured segments. Three slices from each well were picked and covered with Ibal antibody (019-19741, $1: 1,000$ ) and incubated overnight at room temperature, then Cy3-conjugated anti-rabbit IgG (111-165-003, 1:400) and Dapi were added and incubated at room temperature for 1 hour. Slices with the strongest signal contrast between the left and right sides were chosen, the wells in which they were located were the sections from the injured segments of the spinal cord. These sections are used for follow-up tests.

Single or double labeling method was used for immunofluorescence.

Single-labeling: slices from target sections were incubated with the S100A4 antibody (ab41532, 1:50) overnight at room temperature, and then incubated separately with FITC-conjugated anti-rabbit IgG (111-095-003, 1:400) or Cy3-conjugated anti-rabbit IgG (111-165-003, 1:400) and Dapi at room temperature for 1 hour. Slices were washed by PBST three times (10 minutes for each) after every incubation, observation and photo-taken were conducted by a fluorescence microscope.

Double-labeling: The CGRP antibody (ab36001, 1:100) and the RAGE antibody (AF1145, 1:200) were added to slices stained with S100A4 (green signal) overnight. Cy3-conjugated donkey anti-goat $(1: 400)$ was then added and incubated for 1 hour.

Since the antibodies of Iba1 (019-19741) and P2X3 (APR-016) belong to the same species as the S100A4 antibody, Tyramide Signal Amplification (TSA) fluorescein system was used to detect the co-labeling of S100A4 with the above molecules. ${ }^{20,21}$ Slices were incubated with the S100A4 antibody (1:400) overnight at room temperature and then incubated with biotin-conjugated donkey anti-rabbit IgG (711-625-152, 1:500) and DAPI at room temperature for 1 hour. Then slices were incubated with SA-HRP (1:500) at room temperature in darkness for 30 minutes and then incubated with $0.03 \% \mathrm{H}_{2} \mathrm{O}_{2}$ and tyramine-conjugated fluorescein (Cy3 or FITC, 1:100 diluted with PBS) at room temperature in darkness for 10 minutes. Thereafter the secondary primary antibody incubation was performed. Slices was incubated with AntiP2X3 (1:1,000), anti-Iba1 (1:400), biotin-conjugated-IB4 (1:400) overnight at room temperature, respectively, then were incubated with FITC- or Cy3-conjugated donkey anrabbit IgG (diluted 1:400) at room temperature in darkness for 1 hour. Slices were washed with PBST three times (10 minutes for each) after every incubation, Slices were observed with a fluorescence microscope (EclipseE600, Nikon, Japan), and photos were taken by a digital camera (DXM1200, Nikon, Japan) attached to the microscope.

\section{Western Blot}

The spinal cord and DRG were washed with PBS, weighed and then cut into small pieces and ground. Cleavage was added with an appropriate amount of RIPA lysate $\left(4^{\circ} \mathrm{C}\right.$, mixed with PMSF) and homogenate was centrifuged at $12,000 \mathrm{rpm}$ for 5 minutes. Supernatant was collected and added with $5 \times$ Loading Buffer (4:1) and then boiled in a $95^{\circ} \mathrm{C}$ water bath for 5 minutes. Electrophoresis was conducted with $12 \%$ SDS-PAGE gel and polyvinylidene fluoride (PVDF) membrane was used. The membrane was blocked in $10 \%$ skim milk and shaken at $25^{\circ} \mathrm{C}$ for 2 hours. After washing with PBST, the S100A4 rabbit antibody $(1: 1,000)$ or GAPDH mouse antibody $(39-8,600$, $1: 20,000)$ was added to the PVDF membrane and incubated overnight at $4^{\circ} \mathrm{C}$. On the next day, the membrane was washed with PBST and then incubated with horseradish peroxidase-conjugated goat anti-rabbit IgG (A0208, $1: 2,500)$ or goat anti-mouse $\operatorname{IgG}(\mathrm{A} 0216,1: 2,500)$ at room temperature for 2 hours. PBS was used to wash the membrane three times (10 minutes for each). Finally, exposure was conducted with ECL developer and photographed with BIO-RAD Molecular Imager instrument. The fluorescence intensity was analyzed by Image $2 \mathrm{x}$ software.

\section{Co-Immunoprecipitation (Co-IP)}

Fresh tissue samples were weighed, rapidly frozen in liquid nitrogen, added with RIPA Lysis Buffer (4-times the tissue weight, containing $1 \% \mathrm{PMSF}$ ) and fully ground. The mixture was transferred into a clean EP tube and placed in the mixer to react at $4{ }^{\circ} \mathrm{C}$ for 30 minutes and then centrifuged at $12,000 \mathrm{~g}$ for 5 minutes. The supernatant was divided into three new EP tubes and then added with S100A4 antibody, RAGE antibody, and inactive rabbit IgG, respectively, with a concentration of 1 $\mu \mathrm{g} / \mathrm{mL}$. These EP tubes were shaken in a shaker overnight at $4^{\circ}$ 
C. On the next day, tubes were added with $20 \mu \mathrm{L}$ of fully suspended Protein A+G Agarose (Beyotime, P2019), shaken slowly on the decolorizing shaker at $4^{\circ} \mathrm{C}$ for 3 hours, and then centrifuged at 2,500 rpm for 5 minutes. Supernatant was carefully discarded, and precipitation was added with $1 \mathrm{~mL}$ of cold $\operatorname{PBS}(0.05 \mathrm{~mol} / \mathrm{L})$ and centrifuged five times (each at 2,500 rpm for 1 minutes). After the last washing, the supernatant was discarded, and the precipitation was fully re-suspended with 20 $\mu \mathrm{L}$ of $1 \times$ SDS-PAGE sample loading buffer and centrifuged at high-speed for a couple of seconds. Samples were boiled at $95^{\circ} \mathrm{C}$ for 5 minutes. After cooling, electrophoresis was performed immediately, otherwise samples were stored at $-20^{\circ} \mathrm{C}$.

\section{Statistics}

All results of the experiments were repeated at least three times independently. Graphpad 8 and SPSS 21 were used for statistical analysis. In the immunofluorescence experiment, the non-operative side was used as control, at least three rats in each group were analyzed, and at least three slices were randomly selected from each part of the tissue of each rat for observation and statistical analysis.

First, data were tested for normal distribution and homogeneity of variance. If the above two conditions were met, data from each group were compared by one-way analysis of variance (one-way ANOVA). Behavioral data were compared by repeated measurement analysis of variance, pairwise comparison was conducted by Dunnett's T3, Holm-Sidak or Tukey post hoc test, and results were presented as mean \pm standard deviation. If the normal distribution is uneven, the independent samples were tested by Brown-Forsythe test. If the above two conditions were both not met, Kruskal-Wallis $H$-test was used and results were presented as median value. The difference was considered as statistically significant if $P<0.05$.

\section{Results}

\section{The Expression of SI00A4 in the Dorsal Root Ganglion and Spinal Cord of SNL Rats}

Changes of S100A4 expression in the dorsal root ganglion after SNL operation was verified by Immunofluorescence. There was no significant difference between the expression level on the 3rd, 7th, and 14th days after SNL compared with that before. Because the variance was uneven, the numbers of positive cells were analyzed by BrownForsythe test, and the difference among the samples was statistically significant. Further pairwise comparison with Dunnett's T3 method showed that there was no significant difference between the numbers of positive cells on the $3 \mathrm{rd}$ and 14th days after SNL $(851.5 \pm 80.1$ vs $862.5 \pm 120.8$, $P=0.101 ; 964.8 \pm 172.9$ vs $862.5 \pm 120.8, P=0.078$, respectively) compared with that before, only the number of S100A4 positive cells at 7 th days after modeling was significantly increased compared with that before $(1,088$ \pm 136.4 vs $862.5 \pm 120.8, P=0.004$ ) (Figure 1).

For rat spinal cord sections, the sections with obvious changes were selected by inflammatory marker Iba1 before conducting double-labeling immunofluorescence. Results showed that S100A4 was mainly expressed in the substantia gelatinosa of the spinal dorsal horn in the lumbar section of the spinal cord, and the expression in the operative side (the dominant side of Iba1) was higher than that in the control side (Figure 2).

Immunofluorescence and Western blotting were used to verify the changes of S100A4 expression in spinal dorsal horn with time after the establishment of SNL. Because of the uneven variance, data of immunofluorescence were analyzed by Brown-Forsythe test. Results showed that the difference was statistically significant $(P<0.001)$. Furthermore, Holm-Sidak test analysis of integral optical density showed that the expressions of S100A4 in spinal dorsal horn on the 3rd, 7th, and 14th day after SNL operation were significantly higher than those of the control group (237.8 \pm 7.19 vs 189.4 $\pm 17.8, P=0.049 ; 393.7 \pm 16.8$ vs $189.4 \pm 17.8, P<0.001 ; 497.6$ \pm 36.0 vs $189.4 \pm 17.8, P=0.004$, respectively) (Figure $3 \mathrm{~A}$ ). Because of the uneven variance, data of Western blotting were analyzed by Brown-Forsythe test. Results showed that the difference was statistically significant $(P<0.001)$. Further pairwise comparison with Dunnett's T3 test showed that the expression of S100A4 protein increased significantly on the 7th, 14th, 21st, and 28th day after SNL compared with that before $(0.85 \pm 0.02$ vs $0.18 \pm 0.01, P<0.001 ; 3.31 \pm 0.05$ vs 0.18 $\pm 0.01, P<0.001 ; 5.69 \pm 0.10$ vs $0.18 \pm 0.01, P<0.001 ; 4.33$ \pm 0.05 vs $0.18 \pm 0.01, P<0.001$, respectively) (Figure $3 \mathrm{~B}$ ).

\section{Co-Labeling of SI00A4 and Cellular Markers in the Dorsal Root Ganglion and Spinal Cord of SNL Rats}

In the dorsal root ganglion, double-labeling immunofluorescence suggested that S100A4 was partially co-labeled with Calcitonin gene-related peptide (CGRP), Isolectin B4 (IB4), and Purine receptor P2X3 (P2X3), markers of small cell peptidergic neurons, small cell non-peptidergic neurons, and small cells associated with pain transmission and neuropathic pain, respectively. The specific co-labeling 

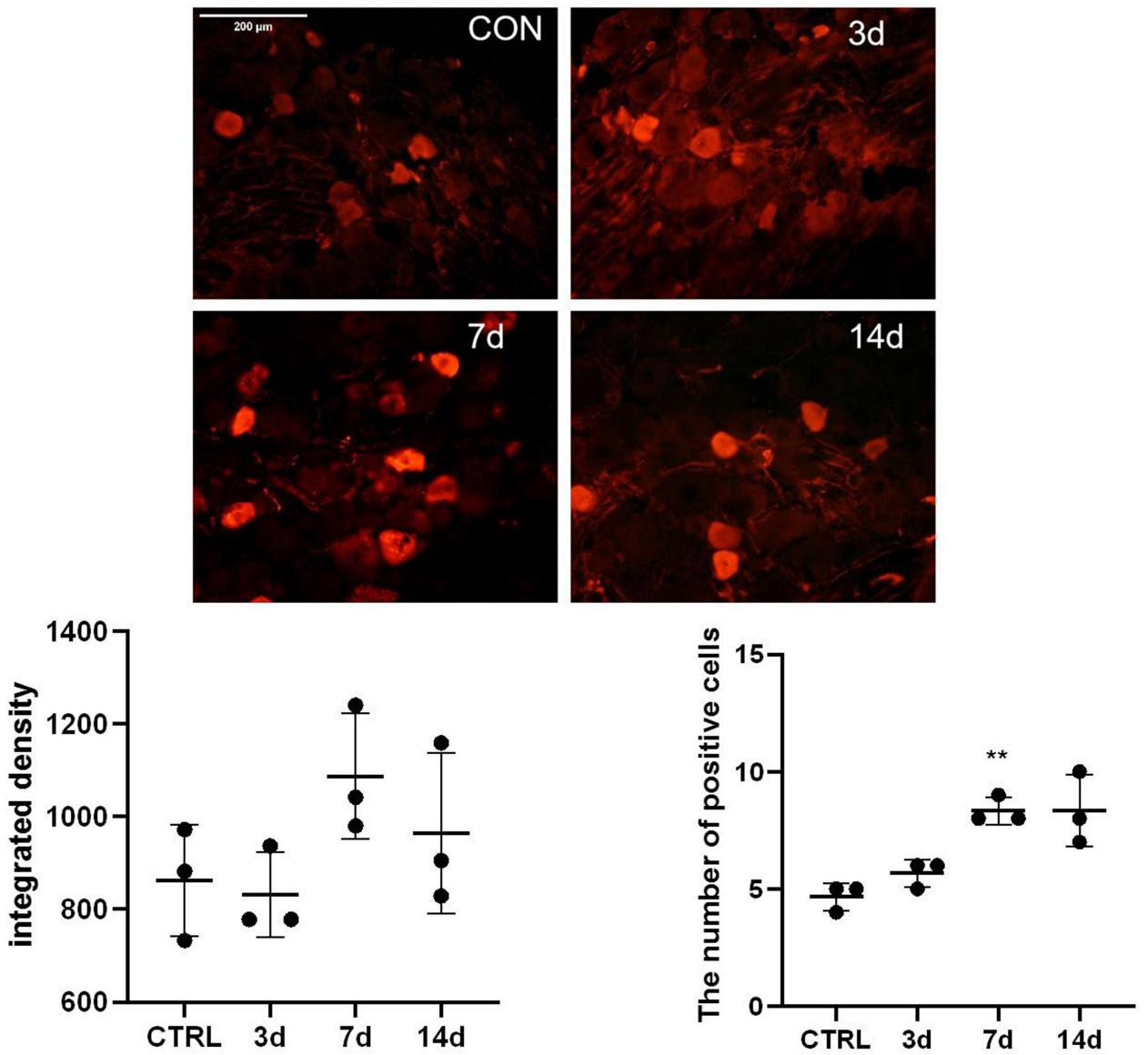

Figure I Expression of SI00A4 in the DRG of SNL rats. After model building, the results of DRG immunofluorescence, the value of integral optical density, and the number of positive cells were compared $(n=3)$. The integral optical density was of no significant difference on the $3 \mathrm{rd}$, 7 th, and 14 th day after SNL compared with that of the control group. The number of positive cells in the SNL group was significantly higher than that of the control group on the 7th day after operation. $* * p<0.01$, bar=200 $\mu \mathrm{m}$.

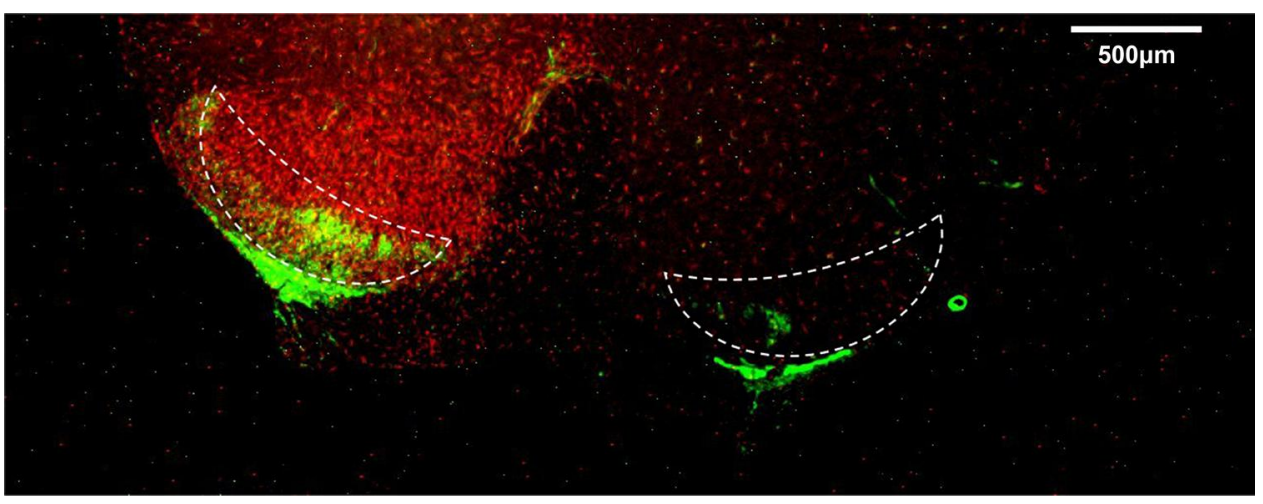

Figure 2 Expression of SI00A4 in the spinal cord of SNL rats. The cross-section of the spinal cord of SNL rats was studied by double-labeling immunofluorescence. The picture shows the merge results of red and green channels. The red fluorescence represented lbal (inflammatory marker), which was brighter on the operation side, and the contralateral side is the control side. The green fluorescence represented SI00A4. The dotted line area was the substantia gelatinosa of the spinal cord dorsal horn where SI 00A4 expressed, and SI00A4 was expressed more on the operation side. The other marginal green fluorescent areas were the white matter of the spinal cord. Ruler $=500 \mu \mathrm{m}$.

proportion was: $62.5 \%$ of IB4 positive cells, $33.3 \%$ of CGRP positive cells, and $50 \%$ of $\mathrm{P} 2 \mathrm{X} 3$ positive cells were co-labeled with S100A4, respectively, whereas the proportion of S100A4 positive cells co-labeled with IB4,
CGRP, and P2X3 were $19.2 \%, 33.3 \%$, and $10 \%$, respectively. The results showed an obvious heterogeneity of S100A4, suggesting that cells with positive S100A4 may be an independent subgroup (Figure 4). 

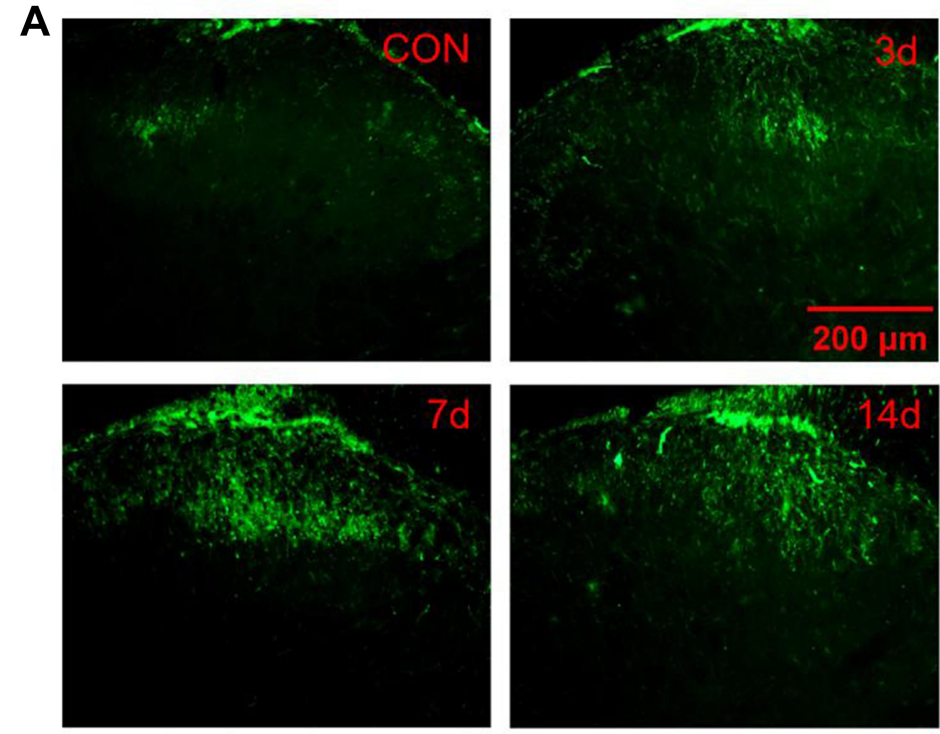

B

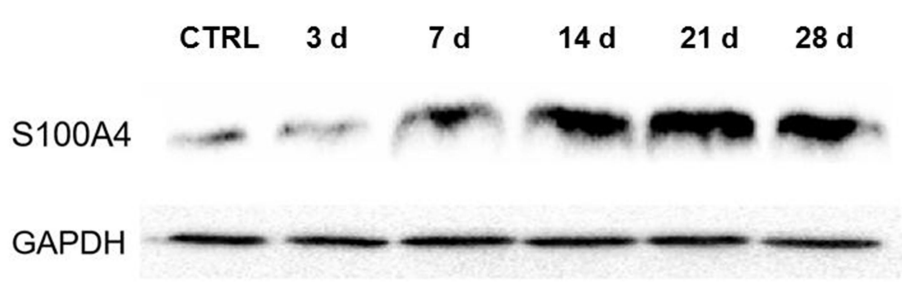

\section{Immunofluorescence}
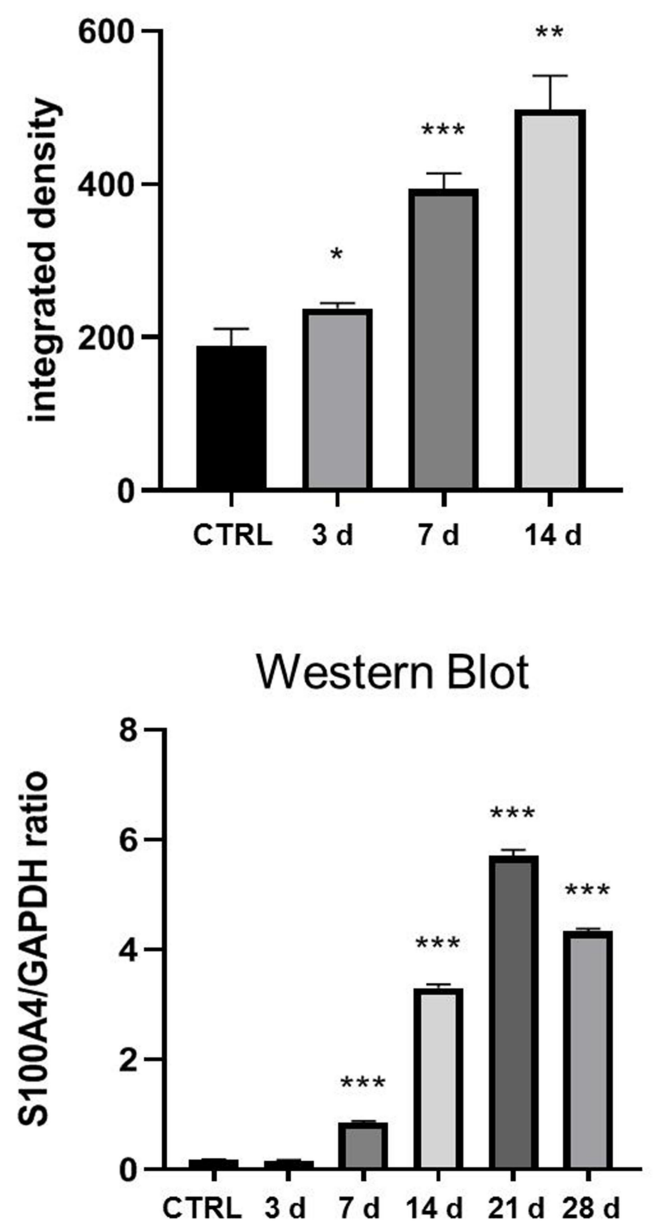

Figure 3 Expression of SI00A4 in the spinal dorsal horn after SNL. (A) Immunofluorescence study of the changes of SI00A4 in spinal dorsal horn after SNL. The fluorescence intensity of SI00A4 increased significantly on the $3 \mathrm{rd}, 7$ th, and I4th day after SNL compared with that of the control group $(* P<0.05$, $* * P<0.0 \mathrm{I}$, $* * * P<0.00 \mathrm{I}$ ). Green: FITC, bar $=200 \mu \mathrm{m}$. (B) WB analyze of expression of SI00A4 in the spinal dorsal horn after SNL. GAPDH was used as internal reference. The expression level of SI00A4 increased significantly on the 7th, 14th, 2 Ist, and 28th day after SNL, compared with that of the control group ( $* * * P<0.00 \mathrm{I}$ ).

In the spinal dorsal horn, results showed that S100A4 was partially co-labeled with Glial fibrillary acidic protein (GFAP), IB4, and Ionized calcium binding adaptor molecule 1 (Iba1), markers of activating astrocytes, primary afferent nerve fibers, and microglia, respectively (Figure 5).

\section{Influence of PTW by Neutralization Antibody Intervention in Rat SNL Model}

Repeated measurement analysis of variance data was performed and the sphericity test showed $P=0.001$. The Greenhouse-Geisser test results showed that the interaction between intervention and time was significant $(P<0.001)$ (Figure 6; Table 1). Compared with the control group, the PWT of the SNL/saline group was significantly decreased on the 1st $(P<0.003)$, 3rd, 5th, and 7th (all $P<0.001)$ day after SNL operation, and the PWT of the SNL/antibody group was not significantly decreased on the 1st day after SNL operation but was significantly decreased on the 3rd, 5th, and 7th day after $(P<0.007,0.008$, and 0.001 , respectively). At the same time, the PWT of the SNL/antibody group was significantly higher than that of the SNL/saline group on the 3rd, 5th, and 7th day after operation (all $P<0.001$ ). These results indicate that intrathecal injection of S100A4 antibody could increase the mechanical pain threshold on the 3rd, 5th, and 7th day after SNL operation.

\section{The Changes of SI00A4 Expression in Each Group After Intervention}

Western blotting was used to determine the content of S100A4 in the dorsal root ganglion and spinal cord of SNL 


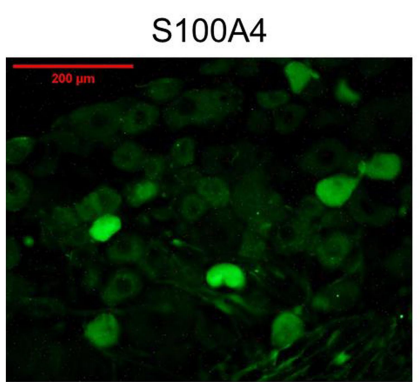

S100A4

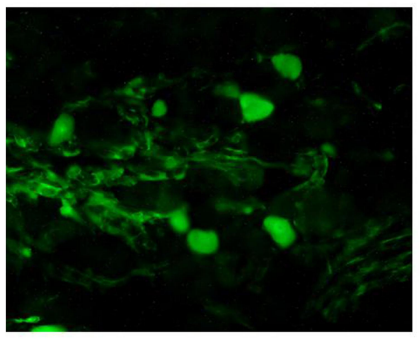

S100A4

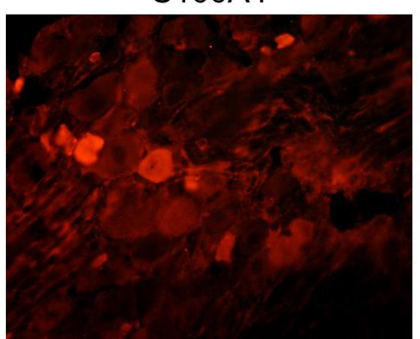

IB4

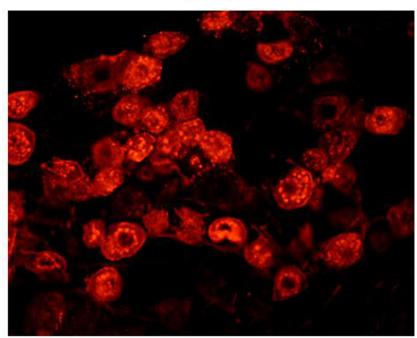

CGRP

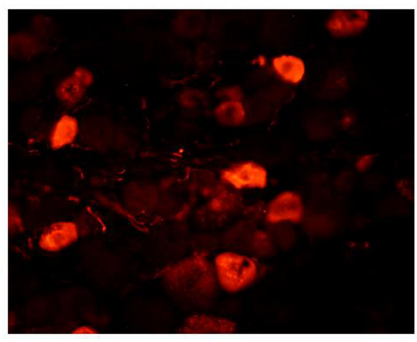

$\mathrm{P} 2 \mathrm{X} 3$

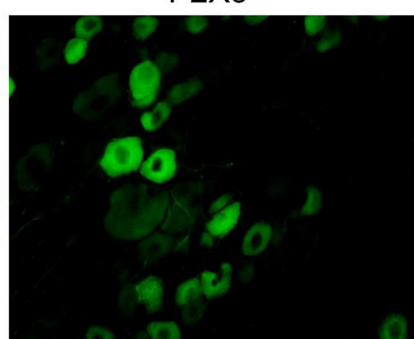

Merge

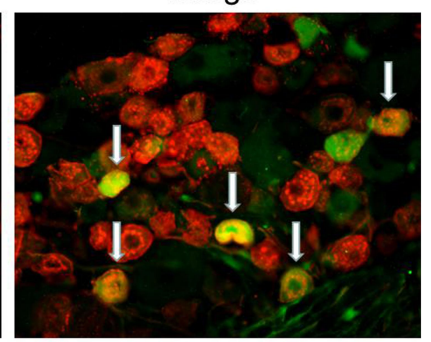

Merge

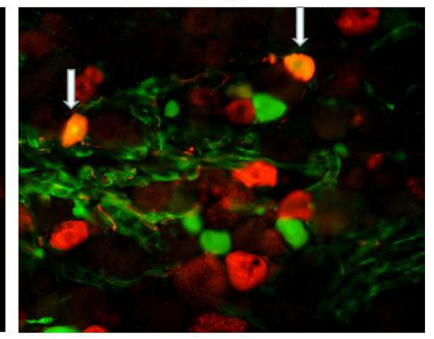

Merge

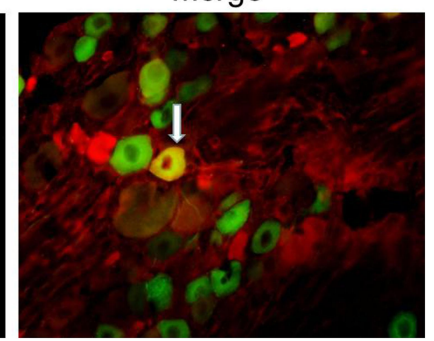

Figure 4 Co-labeling of SI00A4 and certain cell markers in DRG of SNL rats. SI00A4 co-labeled with IB4, CGRP, and P2X3 in the DRG of SNL rats. Results showed that SI00A4 was partially co-labeled with the three markers (the specific co-labeling proportion was: $62.5 \%$ of IB4 positive cells, $33.3 \%$ of CGRP positive cells, and $50 \%$ of P2X3 positive cells were co-labeled with SIO0A4, respectively, whereas the proportion of SIO0A4 positive cells co-labeled with IB4, CGRP and P2X3 were 19.2\%, 33.3\%, and I0\%, respectively). White arrows in the pictures refer to the common label area. Bar=200 $\mu \mathrm{m}$.

rats in each group after intrathecal intervention. Because of the uneven variance, Brown-Forsythe test was used and results showed that the difference was statistically significant $(P<0.001)$. Holm-Sidak test showed that, compared with the control group, the amount of S100A4 protein in the DRG of SNL/antibody group $(0.5223 \pm 0.0750$ vs $0.2477 \pm 0.0160$, $P=0.020)$ and $\mathrm{SNL} /$ saline group $(0.8769 \pm 0.0510$ vs 0.2477 $\pm 0.0160, P=0.003)$ were significantly higher. Besides, the amount of S100A4 protein in the DRG of SNL/antibody group was significantly less than that of the SNL/saline group $(0.5223 \pm 0.0750$ vs $0.8769 \pm 0.0510, P=0.008)$ (Figure 7A). Compared with the control group, the amount of S100A4 protein in the spinal cord of SNL/antibody (0.5701 \pm 0.0375 vs $0.2840 \pm 0.0358, P<0.001)$ and $\mathrm{SNL} /$ saline group $(1.1620 \pm 0.0535$ vs $0.2840 \pm 0.0358, P<0.001)$ were also significantly higher. Similarly, the amount of S100A4 protein in the spinal cord of the SNL/antibody group was significantly lower than that of the $\mathrm{SNL} /$ saline group $(0.5701 \pm 0.0375$ vs $1.1620 \pm 0.0535, P<0.001$ ) (Figure 7B). These results suggested that intrathecal injection of the S100A4 antibody could reduce the content of S100A4 in the DRG and spinal cord of SNL rats.

\section{Interaction Between SI00A4 and RAGE in Rats}

There are many target proteins of S100A4, especially those related to inflammation. After reviewing the literature, several downstream molecules of S100A4 that may be related to pain modulation were screened out, namely RAGE, TLR4, and IL-10R. The interaction of all three molecules with S100A4 were analyzed, with credible results only seen in RAGE, thus only results of experiments on interactions between RAGE and S100A4 were provided.

co-IP was carried out in order to confirm the direct interaction between S100A4 and RAGE. Results showed that S100A4 and RAGE could bind directly and interact with each other (Figure 8A). At the same 


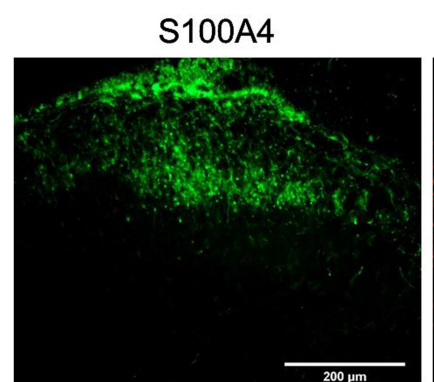

S100A4

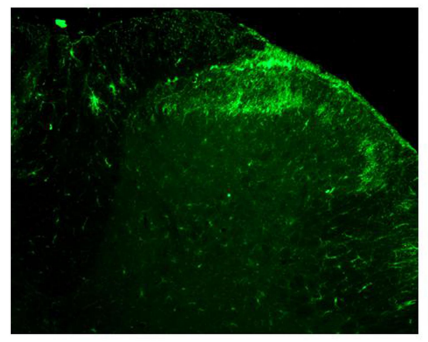

S100A4

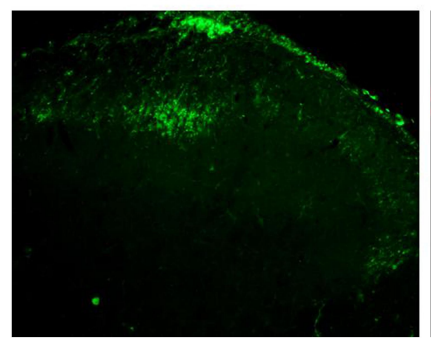

Iba1

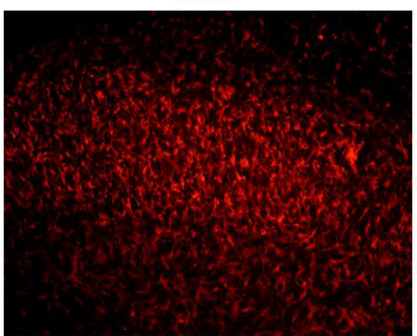

GFAP

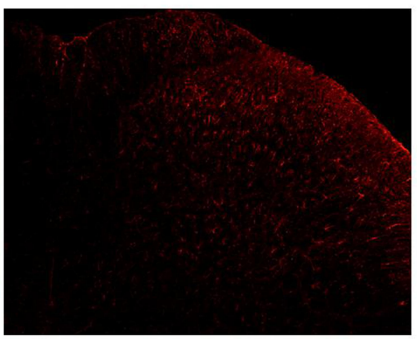

IB4

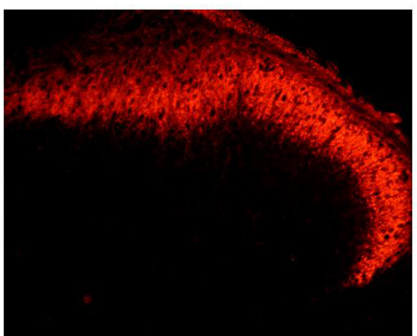

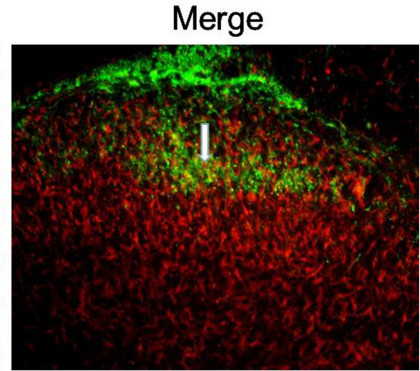

Merge

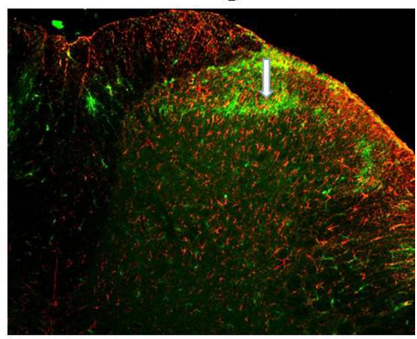

Merge

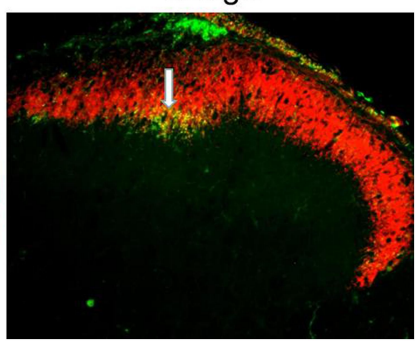

Figure 5 Co-labeling of SIO0A4 and certain cellular markers in spinal dorsal horn of SNL rats. SI00A4 co-labeled with Iba I, GFAP, and IB4 in the spinal dorsal horn of SNL rats. Results showed that SI00A4 was partially co-labeled with the three markers. White arrows in the picture refers to the common label area. Bar=200 $\mu$ m.
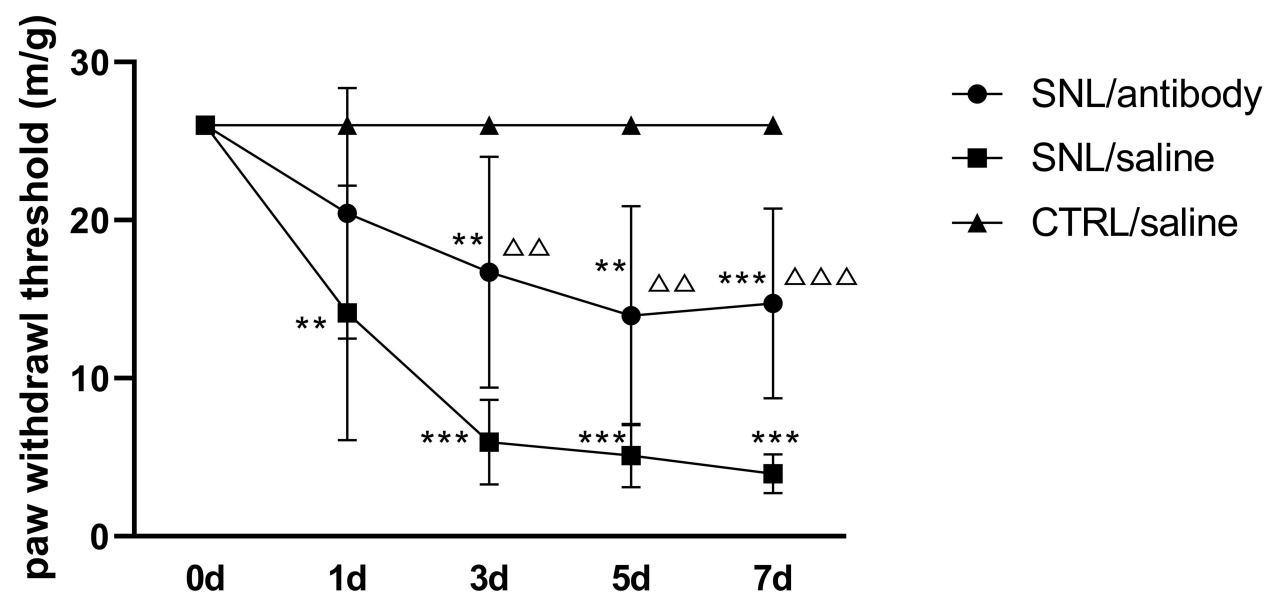

Figure 6 Changes of paw withdrawal threshold in each group after intrathecal intervention. The changes of PWT in each group were measured before and on the Ist, 3rd, 5 th, and 7 th day after SNL operation. Compared with the SNL/saline group, intrathecal injection of SI00A4 neutralizing antibody could increase the PWT on the 3rd, 5th, and 7 th day after operation. ${ }^{* *} P<0.0 \mathrm{I}$, and $* * * P<0.00 \mathrm{I}$, compared with $\mathrm{CTRL} /$ saline; $\Delta \Delta P<0.0 \mathrm{I}$ and $\Delta \Delta \Delta P<0.00 \mathrm{I}$, compared with $\mathrm{SNL} /$ saline. $\mathrm{N}=10$.

time, an immunofluorescence study showed that These results suggested that S100A4 and RAGE interS100A4 and RAGE obviously co-labeled in the sub- acted with each other in the spinal dorsal horn of SNL stantia gelatinosa of spinal dorsal horn (Figure $8 \mathrm{~B}$ ). rats. 
Table I Paw Withdraw Threshold (PWT) in Each Group After Intervention of SNL Model in Rats

\begin{tabular}{|l|c|c|c|c|c|}
\hline & $\begin{array}{c}\mathbf{0} \\
\text { day }\end{array}$ & I day & $\begin{array}{c}\mathbf{3} \\
\text { days }\end{array}$ & $\begin{array}{c}\mathbf{5} \\
\text { days }\end{array}$ & $\begin{array}{c}\mathbf{7} \\
\text { days }\end{array}$ \\
\hline Control group (CON) & 26 & 26 & 26 & 26 & 26 \\
\hline $\begin{array}{l}\text { Placebo group } \\
\text { (SNL/salt) }\end{array}$ & 26 & $\begin{array}{c}14.15 \\
\pm 7.64\end{array}$ & $\begin{array}{c}5.96 \\
\pm 2.55\end{array}$ & $\begin{array}{c}5.12 \\
\pm 1.91\end{array}$ & $\begin{array}{c}3.97 \\
\pm 1.16\end{array}$ \\
\hline $\begin{array}{l}\text { Experimental group } \\
\text { (SNL/antibody) }\end{array}$ & 26 & $\begin{array}{r}20.43 \\
\pm 7.54\end{array}$ & $\begin{array}{c}16.72 \\
\pm 6.92\end{array}$ & $\begin{array}{c}13.95 \\
\pm 6.57\end{array}$ & $\begin{array}{c}14.75 \\
\pm 5.69\end{array}$ \\
\hline
\end{tabular}

\section{Discussion}

$\mathrm{S} 100 \mathrm{~A} 4, \mathrm{Ca}^{2+}$-binding protein, is a member of the $\mathrm{S} 100$ protein family and plays an important role in promoting cancer progression and metastasis. Besides its role in tumors, S100A4 is also involved in a variety of pathophysiological processes in vivo. In the nervous system, the study of S100A4 is mainly focused on neuroprotection and plasticity regulation, such as induction of synaptic proliferation, ${ }^{11}$ increase of Akt signal transduction to reduce apoptosis, ${ }^{12}$ up-regulation of multiple antioxidant enzymes and neuroprotective genes, ${ }^{13,14}$ and release of neuroprotective agents. ${ }^{10}$ Through a literature search, we found that S100A4 is a molecule with many functions, which is closely related to inflammation, tumor invasion, and nerve injury repair. So far, no literature on pain modulation of S100A4 has been found, Our question is whether there is a certain relationship between S100A4 and the generation and modulation of neuropathic pain or not. In this study, a classical SNL pain model was used to study the spatio-temporal expression of S100A4. Immunofluorescence and Western blotting proved that the S100A4 protein increased in the substantia gelatinosa of spinal dorsal horn of SNL rats. Mechanical pain threshold measurement showed that intrathecal injection of S100A4 neutralization antibody could relieve the mechanical pain of SNL rats. A co-IP study proved that there was a direct interaction between S100A4 and RAGE.

From the first day after the birth of rats, S100A4 was expressed in the astrocytes of the white matter of brain and spinal cord, the expression level of which decreases in adult rat's central nervous system. ${ }^{18}$ In the SNL rats, S100A4 gathers mainly in the substantia gelatinosa of the dorsal horn in the gray matter of the spinal cord, specifically layer II of the dorsal horn. This area has many intermediate neurons which mainly function in regulating the afferent pain signals from the peripheral receptors to the central nervous system and plays an important neurophysiological role in the gate control theory of pain. This structure receives and integrates primary afferent fibers (PAF) from DRG. Different types of PAF transmit sensory information to substantia gelatinosa, the most abundant of which are $\mathrm{C}$ fibers (transmits information of slow pain, dull pain, heat, and itching), A- $\delta$ fibers (severe pain and cold), and A- $\beta$ fibers (coarse tactile and mechanical sensation). ${ }^{25} \mathrm{~S} 100 \mathrm{~A} 4$ is abundant in the substantia gelatinosa of spinal dorsal horn, an important part of pain modulation and transmission. All of this knowledge supported that S100A4 could be involved in the generation and modulation of neuropathic pain in a SNL model. Immunofluorescence of spinal cord sections showed that there was no obvious overlap between S100A4 and markers of glial cells, neurons, and other major cells in the spinal glia, and the proportion of S100A4 positive cells among all co-labeled cells was low. This indicated that the S100A4 observed in the dorsal horn of the spinal cord was not expressed by cells in situ, but could be transported here from a distance. The substantia gelatinosa of the dorsal horn of the spinal cord is the projection site of the primary afferent fibers of DRG. It has been proved that S100A4 can act on other cells through paracrine and autocrine. ${ }^{26}$ Immunofluorescence and Western blotting confirmed that S100A4 was expressed in the DRG, thus, the S100A4 in the substantia gelatinosa of spinal dorsal horn may be secreted by DRG cells and transported to the spinal dorsal horn through PAF, where it acts in the form of exocrine protein. The number of S100A4 positive cells showed by immunofluorescence increased significantly only on the 7 th day after SNL operation. There was no significant difference in integral density between the SNL group and the control group, however Western blotting showed a significant difference in protein content. The reason may be that S100A4 protein expressed by DRG cells did not remain in the cytoplasm after assembly but directly entered the axoplasmic transport, thus, the intracellular concentration of which was not increased but distributed more in the nerve fibers until being transported to the dorsal horn of the spinal cord. In the DRG of SNL rats, S100A4 positive cells were partially co-labeled with CGRP, IB4, and P2X3, of which CGRP is a specific marker of small cell peptidergic neurons which are thought to mainly transmit pain sensitization caused by inflammatory response, IB4 is a specific marker of small cell non-peptidergic neurons which are thought to transmit pain caused by nerve 


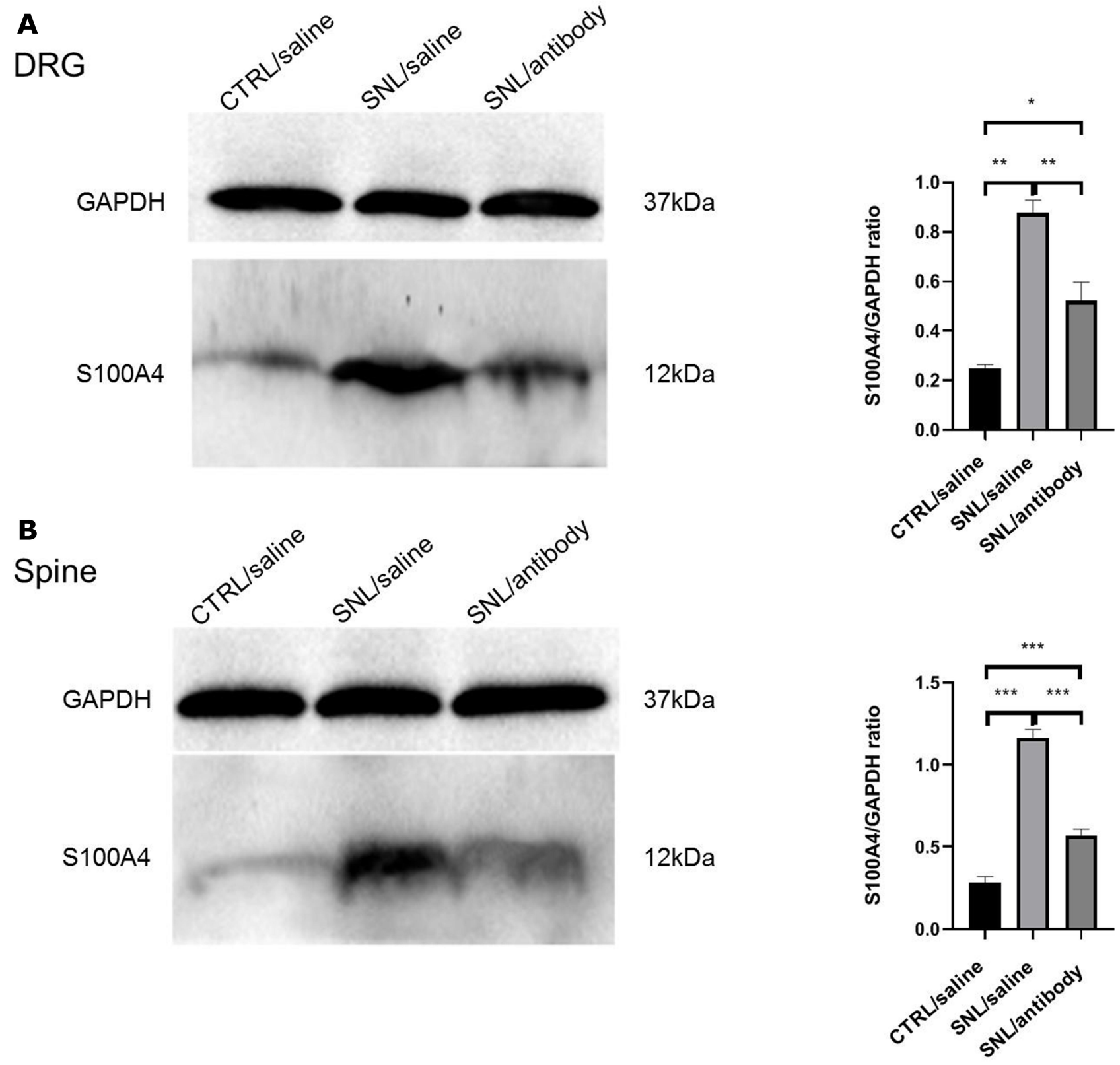

Figure 7 Changes of SI00A4 expression in each group after intrathecal intervention. Western blotting results of SI00A4 expression changes in each group after intrathecal intervention. GAPDH was used as internal reference. (A) The expression of SI00A4 in the DRG of the three groups. The expression level of SI00A4 in the DRG of SNL/ antibody and SNL/saline groups were significantly higher than that of the CTRL/saline (control) group. Besides, the expression level of SI00A4 in the DRG of the SNL/ antibody group was significantly lower than that of the SNL/saline group. (B) The expression of SI00A4 in the spinal cord of the three groups. The expression level of SI00A4 in the spinal cord of the SNL/antibody and SNL/saline groups were significantly higher than that of the control group. Similarly, the expression level of SI00A4 in the spinal cord of the SNL/antibody group was significantly lower than that of the SNL/saline group. $* P<0.05$. $* * P<0.01$. $* * * P<0.001$.

damage, and $\mathrm{P} 2 \mathrm{X} 3$ is an ionic purine receptor in response to extracellular ATP and has been confirmed to be highly expressed in the small and medium-sized nociceptors of DRG, marking small cells related to pain transmission and neuropathic pain. $^{27}$ These three kinds of DRG neurons are all important parts in the pathways of pain generation and transmission. The partial co-labeling of S100A4 with these three markers indicates that S100A4 may be involved in pain transmission and modulation. Meanwhile, it is possible that S100A4 positive cells may be a relatively independent group of cells, initiating different mechanisms of pain regulation. In the spinal cord, S100A4 was co-labeled with GFAP, Iba1, and IB4, which are markers of activated astrocytes, microglia, and the projection site of primary sensory fibers in the dorsal horn, respectively. Co-labeling with IB4 indicated that S100A4 was partially distributed in 
A

B
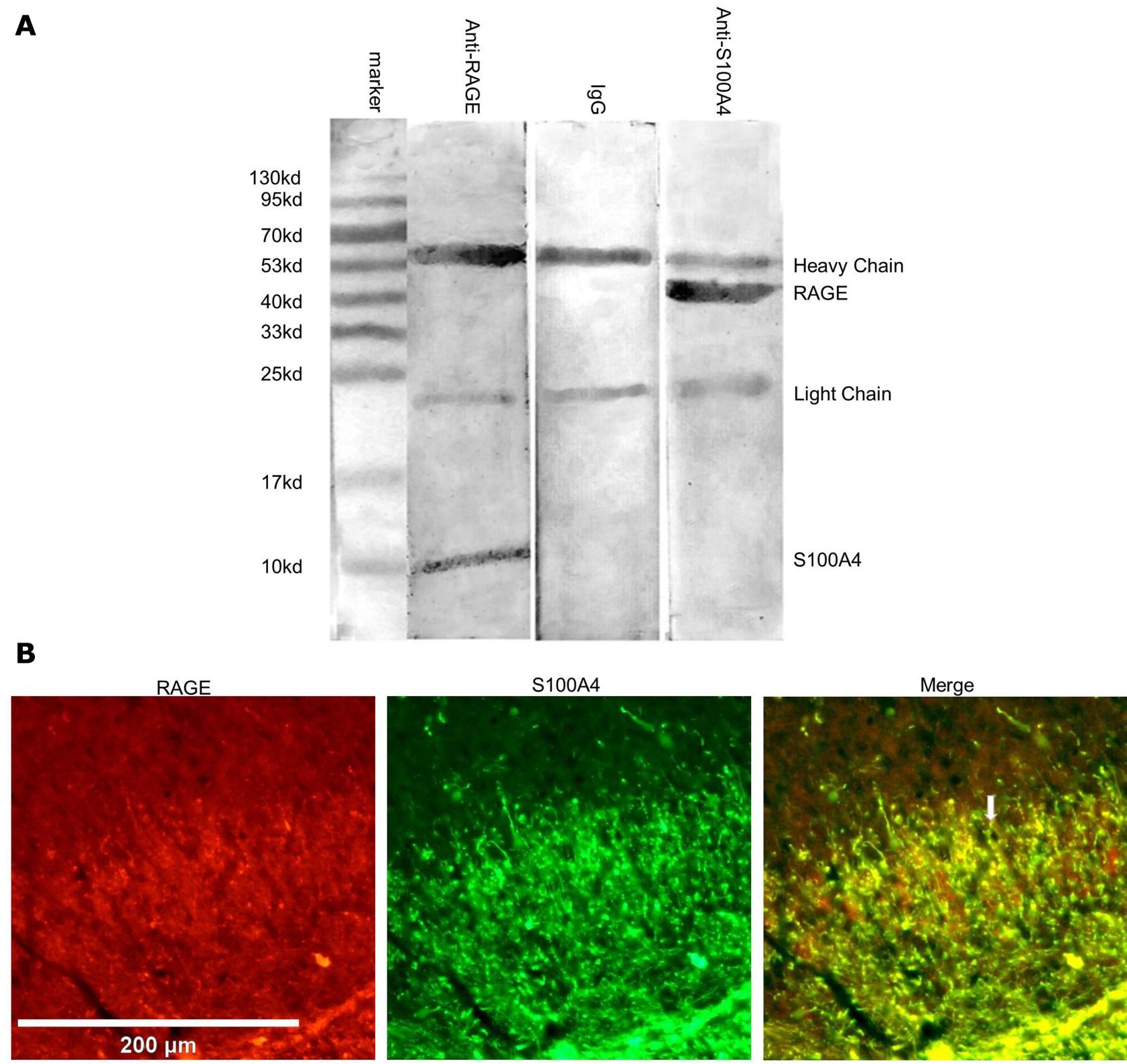

Figure 8 Interaction between SI00A4 and RAGE in rats. (A) Results of co-IP showed that the corresponding molecules could be detected in the immune complexes pulled by SI00A4 or RAGE antibody, while those pulled down by ineffective lgG could not detect these two molecules. (B) Immunofluorescence of the spinal dorsal horn showed that SI00A4 and RAGE co-labeled with each other in the substantia gelatinosa of the spinal dorsal horn. White arrow: hint of co-labeled point, no significant. Red: Cy3. Green: FITC. Scale $=200 \mu \mathrm{m}$.

the peptidergic nerve fibers emitted by DRG, which was consistent with the co-labeling of neuronal subsets observed in DRG. Previous studies have shown that S100A4 is only expressed in the astrocytes of white matter not gray matter. Even after injury, astrocytes of gray matter still do not express S100A4. ${ }^{28}$ Our study showed that the distribution of S100A4 was highly concentrated in the substantia gelatinosa of spinal dorsal horn which was the only site which observed the co-labeling of S100A4 with GFAP and Iba1, however, GFAP and Ibal positive cells were distributed in both the dorsal and ventral horns of the reaction side. We believe that the S100A4 in the spinal cord was transported from the PAF to the substantia gelatinosa and then acted on the astrocytes and microglia in the gray matter of the spinal cord rather than expressed by themselves.

co-IP and immunofluorescence studies proved that there was a direct interaction between S100A4 and RAGE in the dorsal horn of spinal cord. RAGE itself has a wide range of functions and is related to the pathogenesis 
of neurovascular and neurodegenerative diseases. It is expressed by a variety of cell types in the brain and spinal cord, including neurons, glia (microglia and astrocytes), and vascular cells (endothelial cells, smooth muscle cells, and pericytes). ${ }^{29,30}$ Therefore, S100A4 may participate in neuropathic pain by combining with RAGE to assist in activating astrocytes and microglia.

Neuropathic pain is triggered by lesions of the somatosensory nervous system which changes its structure and function, thus pain occurs spontaneously. Meanwhile, the response to harmful or harmless stimuli is pathologically magnified. ${ }^{2}$ At present, the most mainstream views on the mechanism of neuropathic pain believe that neuropathic pain is associated with the role of NMDA receptor, inflammatory mediators, spinal cord opioid, and antagonistic system balance, central sensitization, central disinhibition, and spinal cord glial cell activation. As a multifunctional molecule, S100A4 may exert effects on neuropathic pain on these aspects.

$\mathrm{S} 100 \mathrm{~A} 4$ is a member of damage associated molecular patterns (DAMPs). During normal physiological processes in vivo, DAMPs-mediating inflammation enables the host to remove associated cell fragments and then eliminate inflammation and repair tissue if necessary. However, exaggerated DAMPs signal transduction is the basis of "aseptic inflammation", which is an inflammatory response driven in the absence of obvious infection. ${ }^{31}$ S100A4 is an effective promoter of the inflammatory process and is involved in various links of inflammatory response through multiple pathways. Many receptors of S100A4 are related to inflammation-related signal transduction, such as RAGE receptor, TLR4, and IL-10R. ${ }^{10}$ $\mathrm{S} 100 \mathrm{~A} 4$ is a regulator of physiological macrophage movement, which mediates macrophage recruitment and chemotaxis in vivo through the secretion of various inflammatory factors. This is very important for the recruitment of neutrophils and macrophages to the inflammatory site of the body. Loss of S100A4 can lead to impaired recruitment of macrophages. ${ }^{32}$ In cardiomyocytes, S100A4 increases the synthesis of proinflammatory cytokines in monocytes by autocrine and/or paracrine, and significantly increases the expression and release of IL-1 $\beta$, IL-6, and TNF- $\alpha$ in monocytes. ${ }^{26}$ The release of a large number of inflammatory cytokines can significantly aggravate the degree of pain. S100A4 can activate NF- $\kappa \mathrm{B}$, a main pro-inflammatory transcription factor, by interacting with RAGE and inducing the phosphorylation of Pyk-2 and MAPKs. ${ }^{33,34}$ S100A4 can also activate acute phase reactive protein serum amyloid protein A (SAA) through the signal transduction of the TLR4/NF- $\kappa \mathrm{B}$ pathway, thereby stimulating the transcription of a variety of cytokines and forming a chronic inflammatory environment. ${ }^{35}$ Therefore, S100A4 may be involved in the process of neuropathic pain by regulating nerve-related inflammatory responses. In this study, the changes of related inflammatory factors before and after intervention were not analyzed, and whether S100A4 affects the process of neuropathic pain by regulating inflammatory molecules remains to be confirmed by further experiments.

Central sensitization refers to the abnormal increase in excitability of the spinal cord and brain under the longterm stimulation of inflammatory response and nerve trauma. This sensitization results from the release of excitatory neurotransmitters which activate NMDA and nonNMDA receptors. ${ }^{36}$ It is reported that the RAGE/ STAT3 signal transduction pathway in the dorsal horn was activated, and the spinal cord central sensitization and persistent pain caused by bortezomib treatment aggravated, by the accumulation of methyl Glyoxal. ${ }^{37}$ RAGE pathway is one of the targets of S100A4. In addition, S100A4 can also increase the phosphorylation of extracellular ERK and MAPKs, and activate NF- $\kappa$ B through RAGE-dependent regulation which is related to cell migration and chemotaxis. Phosphorylated ERK has nociceptive specificity and can be used as a marker of central sensitization. S100A4 also acts on immune cells to release IL- $1 \beta^{26}$ and regulates synaptic transmission balance in the spinal cord by enhancing excitatory and weakening inhibitory synaptic transmission. $^{38}$ Therefore, S100A4 may play a painpromoting role in central sensitization and central disinhibition. The detection of NMDA receptor activity is not involved in this study, which awaits further research.

The activation of glial cells and the interaction among different kinds of glia are also key mechanisms of neuropathic pain. RAGE, widely distributed in astrocytes and microglia, provides a possible bridge for S100A4 to interfere with the pain process from extracellular effects to glial cells. Activated microglia in the dorsal horn of the spinal cord are necessary for the synaptic changes in this region and the pain hypersensitivity after nerve injury. Glial cell mediators have been shown to effectively regulate the excitatory and inhibitory transmission, both pre-synaptic, postsynaptic, and extra-synaptic. Therefore, pain may be the result of "gliosis", that is, glial dysfunction in the central and peripheral nervous systems. ${ }^{39}$ Evidence showed that activated astrocytes can release pro-inflammatory cytokines (such as IL-1 $\beta$ ) and chemokines (such as monocyte 
chemoattractant protein-1 [MCP-1]/CCL2) into the spinal cord to enhance and prolong the persistent pain state. ${ }^{38}$ Nerve injury and inflammation activate c-Jun N-terminal kinase $(\mathrm{JNK})$ in the astrocytes of spinal cord and increase the expression and release of $\mathrm{MCP}-1$ which can increase pain sensitivity by directly activating NMDA receptors in dorsal horn neurons. Previous studies have shown that inhibition of IL-1 $\beta$, JNK, MCP-1, or MMP2 signal transduction through spinal cord administration could reduce pain related to inflammation, neuralgia, or cancer. ${ }^{38}$ On the other hand, microglial mediators (such as proinflammatory and anti-inflammatory cytokines) are powerful neuroregulators that regulate synaptic transmission and pain through neuron-glial interactions. ${ }^{40}$ In recent decades, many studies have shown that neuroimmune interaction is the basis for the generation and maintenance of neuropathic pain. The disorder or loss of homeostasis in the central nervous system stimulates a rapid response of microglia, the latter then undergoes a multi-stage activation process. Activated microglia in the spinal dorsal horn are necessary for the synaptic changes in this region and the pain hypersensitivity after nerve injury. ${ }^{41}$ Activated microglia release a large number of inflammatory factors which may act in ways either harmful or beneficial to the surrounding cells and can indirectly alter nociceptive signals. Drug inhibition of microglia proliferation induced by a pain model can weaken neuropathic pain hypersensitivity. ${ }^{42}$ The most important signal cascades in microglia (MAPK, NF- $\kappa$ B, JAK/STAT, PI3K/Akt) are essential for the occurrence and maintenance of neuropathic pain. ${ }^{41}$ The NF- $\mathrm{KB}$ transcription pathway is the major intracellular signal transduction pathway activated by RAGE receptor of astrocytes, which induces the expression and release of proinflammatory cytokines. S100A4 may release pro-inflammatory cytokines from activated microglia and astrocytes through the RAGE$\mathrm{NF}-\kappa \mathrm{B}$ pathway and participate in the occurrence and development of pain. In this study, the activation degree of glial cells before and after neutralization antibody intervention was not compared, which remains to be confirmed by further experiments.

To sum up, RAGE, which can bind to S100A4 in CO-IP test, can activate NF- $\mathrm{KB}$ through the ERK-MARK pathway. This pathway can release a large number of inflammatory factors, or cause central sensitization in the dorsal horn of the spinal cord. At the same time, this pathway can activate a strong signal cascade in glial cells (MAPK, NF- $\kappa B, J A K /$ STAT, PI3K/Akt). These are all possible ways in which the S100A4/RAGE pathway affects neuropathic pain.

\section{Conclusion}

The content of S100A4 in the DRG and spinal dorsal horn increased in SNL rats. Intrathecal injection of S100A4 neutralizing antibody could effectively relieve the mechanical pain in SNL rats. S100A4 may be involved in the production of neuropathic pain through RAGE or other ways, and the specific mechanism needs to be further studied.

\section{Acknowledgments}

The study was supported by the National Natural Science Foundation of China (No. 81971046).

Xin Jiang, Wenqi Zhao, and Tiantian Zhao contributed equally to this work and should be considered as co-first authors.

\section{Disclosure}

The authors declare no potential conflicts of interest with respect to this work, the research, authorship, or publication of this article.

\section{References}

1. Langley PC, Van Litsenburg C, Cappelleri JC, Carroll D. The burden associated with neuropathic pain in Western Europe. $J$ Med Econ. 2013;16(1):85-95. doi:10.3111/13696998.2012.729548

2. Costigan M, Scholz J, Woolf CJ. Neuropathic pain: a maladaptive response of the nervous system to damage. Annu Rev Neurosci. 2009;32(1):1-32. doi:10.1146/annurev.neuro.051508.135531

3. Hu JZ, Rong ZJ, Li M, et al. Silencing of IncRNA PKIA-AS1 attenuates spinal nerve ligation-induced neuropathic pain through epigenetic downregulation of CDK6 expression. Front Cell Neurosci. 2019;13:50. doi:10.3389/fncel.2019.00050

4. Taves S, Berta T, Chen G, Ji RR. Microglia and spinal cord synaptic plasticity in persistent pain. Neural Plast. 2013;2013:753656.

5. Kawasaki Y, Xu ZZ, Wang X, et al. Distinct roles of matrix metalloproteases in the early- and late-phase development of neuropathic pain. Nat Med. 2008;14(3):331-336.

6. Zarpelon AC, Rodrigues FC, Lopes AH, et al. Spinal cord oligodendrocyte-derived alarmin IL-33 mediates neuropathic pain. FASEB $j$. 2016;30(1):54-65.

7. Coraggio V, Guida F, Boccella S, et al. Neuroimmune-driven neuropathic pain establishment: a focus on gender differences. Int $J \mathrm{Mol}$ Sci. 2018;19:1.

8. Santamaria-Kisiel L, Rintala-Dempsey AC, Shaw GS. Calciumdependent and -independent interactions of the S100 protein family. Biochem J. 2006;396(2):201-214.

9. Parekh AB. Store-operated Ca2+ entry: dynamic interplay between endoplasmic reticulum, mitochondria and plasma membrane. J Physiol. 2003;547(Pt 2):333-348.

10. Dmytriyeva O, Pankratova S, Owczarek S, et al. The metastasis-promoting S100A4 protein confers neuroprotection in brain injury. Nat Commun. 2012;3(1):1197. doi:10.1038/ ncomms 2202

11. Kiryushko D, Novitskaya V, Soroka V, et al. Molecular mechanisms of $\mathrm{Ca}(2+)$ signaling in neurons induced by the S100A4 protein. Mol Cell Biol. 2006;26(9):3625-3638. doi:10.1128/MCB.26.9.36253638.2006 
12. Doroudgar S, Quijada P, Konstandin M, et al. S100A4 protects the myocardium against ischemic stress. $J$ Mol Cell Cardiol. 2016;100:54-63. doi:10.1016/j.yjmcc.2016.10.001

13. Sarafian TA, Montes C, Imura T, et al. Disruption of astrocyte STAT3 signaling decreases mitochondrial function and increases oxidative stress in vitro. PLoS One. 2010;5(3):e9532. doi:10.1371/journal. pone.0009532

14. Dziennis S, Alkayed NJ. Role of signal transducer and activator of transcription 3 in neuronal survival and regeneration. Rev Neurosci. 2008;19(4-5):341-361. doi:10.1515/REVNEURO.2008.19.4-5.341

15. Fuller SJ, Sivarajah K, Sugden PH. ErbB receptors, their ligands, and the consequences of their activation and inhibition in the myocardium. J Mol Cell Cardiol. 2008;44(5):831-854. doi:10.1016/ j.yjmcc.2008.02.278

16. Sibilia M, Kroismayr R, Lichtenberger BM, Natarajan A, Hecking M, Holcmann M. The epidermal growth factor receptor: from development to tumorigenesis. Differentiation. 2007;75(9):770-787. doi:10.1111/j.1432-0436.2007.00238.x

17. Zhang KH, Han S, Lu PH, Xu XM. Upregulation of S100A4 after spinal cord transection in adult rats. Acta Pharmacol Sin. 2004;25 (8):1007-1012.

18. Kozlova EN, Lukanidin E. Mts1 protein expression in the central nervous system after injury. Glia. 2002;37(4):337-348. doi:10.1002/ glia. 10045

19. Sandelin M, Zabihi S, Liu L, Wicher G, Kozlova EN. Metastasisassociated S100A4 (Mts1) protein is expressed in subpopulations of sensory and autonomic neurons and in Schwann cells of the adult rat. J Comp Neurol. 2004;473(2):233-243. doi:10.1002/cne.20115

20. Austin PJ, Wu A, Moalem-Taylor G. Chronic constriction of the sciatic nerve and pain hypersensitivity testing in rats. J Vis Exp. 2012;61. doi:10.3791/3393

21. Chung JM, Kim HK, Chung K. Segmental spinal nerve ligation model of neuropathic pain. Methods Mol Med. 2004;99:35-45. doi:10.1385/1-59259-770-X:035

22. Henry JL, Yashpal K, Pitcher GM, Chabot J, Coderre TJ. Evidence for tonic activation of NK-1 receptors during the second phase of the formalin test in the Rat. $J$ Neurosci. 1999;19(15):6588-6598. doi:10.1523/JNEUROSCI.19-15-06588.1999

23. Vincent AM, Perrone L, Sullivan KA, et al. Receptor for advanced glycation end products activation injures primary sensory neurons via oxidative stress. Endocrinology. 2007;148(2):548-558. doi:10.1210/ en.2006-0073

24. Malcangio M. Role of the immune system in neuropathic pain. Scand J Pain. 2019;20(1):33-37. doi:10.1515/sjpain-2019-0138

25. Sheikh NK, Dua A. Neuroanatomy, Substantia Gelatinosa. StatPearls. Treasure Island (FL): StatPearls Publishing StatPearls Publishing LLC; 2020.

26. Cerezo LA, Kuncova K, Mann H, et al. The metastasis promoting protein S100A4 is increased in idiopathic inflammatory myopathies Rheumatology. 2011;50(10):1766-1772. doi:10.1093/rheumatology/ ker218

27. Xiang X, Wang S, Shao F, et al. Electroacupuncture stimulation alleviates CFA-Induced inflammatory pain via suppressing P2X3 Expression. Int $J$ Mol Sci. 2019;20(13):13. doi:10.3390/ ijms20133248

Journal of Pain Research

\section{Publish your work in this journal}

The Journal of Pain Research is an international, peer reviewed, open access, online journal that welcomes laboratory and clinical findings in the fields of pain research and the prevention and management of pain. Original research, reviews, symposium reports, hypothesis formation and commentaries are all considered for publication. The manuscript

Submit your manuscript here: https://www.dovepress.com/journal-of-pain-research-journal
28. Kozlova EN, Lukanidin E. Metastasis-associated mts1 (S100A4) protein is selectively expressed in white matter astrocytes and is up-regulated after peripheral nerve or dorsal root injury. Glia. 1999;27(3):249-258. doi:10.1002/(SICI)1098-1136(199909) 27:3<249::AID-GLIA6>3.0.CO;2-E

29. Gonzalez-Reyes RE, Rubiano MG. Astrocyte s RAGE: more Than Just a Question of Mood. Cent Nerv Syst Agents Med Chem. 2018;18 (1):39-48. doi:10.2174/1871524916999160505105121

30. MacLean M, Derk J, Ruiz HH, Juranek JK, Ramasamy R, Schmidt AM. The Receptor for Advanced Glycation End Products (RAGE) and DIAPH1: implications for vascular and neuroinflammatory dysfunction in disorders of the central nervous system. Neurochem Int. 2019;126:154-164. doi:10.1016/j.neuint.2019.03.012

31. Ellson CD, Dunmore R, Hogaboam CM, Sleeman MA, Murray LA. Danger-associated molecular patterns and danger signals in idiopathic pulmonary fibrosis. Am J Respir Cell Mol Biol. 2014;51(2):163-168. doi:10.1165/rcmb.2013-0366TR

32. Li ZH, Dulyaninova NG, House RP, Almo SC, Bresnick AR. S100A4 regulates macrophage chemotaxis. Mol Biol Cell. 2010;21 (15):2598-2610. doi:10.1091/mbc.e09-07-0609

33. Weatherly K, Bettonville M, Torres D, Kohler A, Goriely S, Braun MY. Functional profile of S100A4-deficient T cells. Immun Inflamm Dis. 2015;3(4):431-444. doi:10.1002/iid3.85

34. Grotterod I, Maelandsmo GM, Boye K. Signal transduction mechanisms involved in S100A4-induced activation of the transcription factor NF-kappaB. BMC Cancer. 2010;10:241. doi:10.1186/14712407-10-241

35. Hansen MT, Forst B, Cremers N, et al. A link between inflammation and metastasis: serum amyloid $\mathrm{A} 1$ and $\mathrm{A} 3$ induce metastasis, and are targets of metastasis-inducing S100A4. Oncogene. 2015;34 (4):424-435. doi:10.1038/onc.2013.568

36. Ji RR, Nackley A, Huh Y, Terrando N, Maixner W. Neuroinflammation and central sensitization in chronic and widespread pain. Anesthesiology. 2018;129(2):343-366. doi:10.1097/ ALN.0000000000002130

37. Wei JY, Liu CC, Ouyang HD, et al. Activation of RAGE/STAT3 pathway by methylglyoxal contributes to spinal central sensitization and persistent pain induced by bortezomib. Exp Neurol. 2017;296:74-82. doi:10.1016/j.expneurol.2017.07.010

38. Gao YJ, Ji RR. Targeting astrocyte signaling for chronic pain. Neurotherapeutics. 2010;7(4):482-493.

39. Ji RR, Berta T, Nedergaard M. Glia and pain: is chronic pain a gliopathy? Pain. 2013;154(Suppl 1):S10-S28.

40. Chen G, Zhang YQ, Qadri YJ, Serhan CN, Ji RR. Microglia in pain: detrimental and protective roles in pathogenesis and resolution of pain. Neuron. 2018;100(6):1292-1311.

41. Inoue K, Tsuda M. Microglia in neuropathic pain: cellular and molecular mechanisms and therapeutic potential. Nat Rev Neurosci. 2018;19(3):138-152.

42. Gu N, Peng J, Murugan M, et al. Spinal microgliosis due to resident microglial proliferation is required for pain hypersensitivity after peripheral nerve injury. Cell Rep. 2016;16(3):605-614. management system is completely online and includes a very quick and fair peer-review system, which is all easy to use. Visit http:// www.dovepress.com/testimonials.php to read real quotes from published authors. 